\title{
A novel weak form quadrature element for gradient elastic beam theories
}

\author{
Md. Ishaquddin*, S. Gopalakrishnan \\ Department of Aerospace Engineering, Indian Institute of Science, Bengaluru 560012, India
}

\section{A R T I C L E I N F O}

\section{Article history:}

Received 4 September 2018

Revised 26 June 2019

Accepted 4 July 2019

Available online 10 July 2019

\section{Keywords:}

Quadrature element

Strain gradient Euler-Bernoulli beam theory

Weighting coefficients

Non-classical degrees of freedom

Hermite interpolation

\begin{abstract}
A B S T R A C T
A novel weak form quadrature element is proposed for non-classical strain gradient EulerBernoulli beam theories. The element is formulated with the aid of variational principles and has displacement as the only degree of freedom in the element domain and displacement, slope and curvature at the boundaries. All the classical and non-classical support conditions associated with the gradient beam theory are represented accurately. The Gauss-Lobatto-Legendre quadrature points are considered as element nodes and also used for numerical integration of the element matrices. Numerical examples on bending, free vibration and stability analysis of gradient beams are presented to demonstrate the efficiency and accuracy of the proposed element. To substantiate the generality of the element, beams with discontinuity in loading and geometry are examined.
\end{abstract}

(c) 2019 Elsevier Inc. All rights reserved.

\section{Introduction}

In recent decades the research in the field of computational solid and fluid mechanics focussed on developing cost effective and highly accurate numerical schemes. Subsequently, many numerical schemes were proposed and applied to various engineering problems. The early research emphasized on the development of finite element [1-3] and finite difference methods $[4,5]$, these methodologies had limitations related to the computational cost [6]. Alternatively, differential quadrature method (DQM) was proposed by Bellman et al. [7], which employs less number of grid points [8] and quantifies the derivative at a particular grid point as a weighted linear sum of the function values at all grid points in the domain. Later, many enriched versions of differential quadrature method were developed, for example, differential quadrature method [9-12], harmonic differential quadrature method [13], strong form differential quadrature element method (DQEM) [8,14,15], local adaptive differential quadrature method (La-DQM) [16], generalized differential quadrature rule (GDQR) [17,18] and weak form quadrature element method [19-26]. The main theme in these improved DQ versions was to develop versatile models to account for complex loading, discontinuous geometries and generalized boundary conditions.

Lately, much research inclination is seen towards the development of weak form quadrature element methods due to their versatility and high computational efficiency [19-28]. The weak form quadrature element method is formulated using variational principles. The integrands associated with the weak form expression are first approximated using the assumed interpolation function and the corresponding weighting coefficients are evaluated at the sampling points using differential quadrature rule. Later, the weighting coefficients are numerical integrated to form the element matrices $[19,23,28]$. The numerical integration scheme forms an integral part of the weak form quadrature element method as it determines the

\footnotetext{
* Corresponding author.

E-mail addresses: ishaquddinmd@iisc.ac.in (Md. Ishaquddin), krishnan@iisc.ac.in (S. Gopalakrishnan).
} 
number of nodes and facilitates in constructing the higher order elements. A comprehensive survey on the developments and recent contributions in the field of quadrature element method and its application to structural mechanics problems can be found in Wang et al. [19]. Zhong et al. [20] presented a weak form quadrature element for free vibration analysis of eccentric annular Mindlin plates and demonstrated an excellent agreement with the literature results. Further, Zhong et al. extended its application to various structural mechanics problems, for example, planar elasticity problems [21], static and free vibration analysis of planar frameworks [22], static, free vibration and stability analysis of beams based on the Bickford beam theory [23], stability analysis of planar frameworks [24] and analysis of thin plates [25]. Recently, Wang et al. [26] proposed a weak form quadrature element which uses expanded Chebyshev points as element nodes and illustrated its effectiveness for free vibration analysis of bars and beams. Further, Wang et al. [27] presented a discrete singular convolution element and a quadrature beam/ring element for free vibration analysis of non-local beams and non-uniform rings. Liao et al. [28] presented a weak form quadrature element for a three dimensional beam for non-linear analysis of space frames.

The main theme of this article is to present a weak form quadrature element for non-classical higher order beam theories [29]. The non-classical higher order beam theories, unlike classical continuum theories, are governed by sixth order differential equation [30]. These non-classical continuum theories are modified versions of classical continuum theories incorporating higher order gradient terms in the constitutive relations. The higher order terms consists of stress and strain gradients accompanied with the intrinsic length scale parameters which account for micro and nano scale effects. These scale dependent non-classical theories are efficient in capturing the micro and nano scale behaviour of structural systems [31-33]. One such class of non-classical gradient elasticity theory is the simplified theory by Mindlin et al. [34], with one gradient elastic modulus and two classical Lame' constants for structural applications [35,36]. This simplified theory was applied earlier to study the static, dynamic and buckling behaviour of gradient elastic beams by deriving the analytical solutions [37-40]. Pegios et al. [41] derived the stiffness matrix by assuming the exact solution to the governing equation as interpolation function for the displacement field. As a result, the obtained stiffness matrix and the structural behaviour were exact. To solve the examples with discontinuity in loading and geometry they have used exact stiffness matrix for each segment of the beam and employed the finite element (FE) framework for assembly. As the stiffness matrix is exact, the solutions obtained using one element for prismatic beams and two elements for non-prismatic beams are exact. Using the exact stiffness matrix and FE frame work they have studied the static and stability analysis of prismatic and non-prismatic gradient elastic beams.

In this paper, we propose a novel version of weak form quadrature element for strain gradient Euler-Bernoulli beam theory, which is characterized by sixth order differential equation and has displacement, slope and curvature as degrees of freedom. The proposed element employs Hermite interpolation as test function and it is formulated with the aid of variational principles, differential quadrature rule and Gauss-Lobatto-Legendre (GLL) quadrature rule. The GLL points are used as element nodes and also to perform numerical integration of element matrices. The proposed element has displacement, slope and curvature as the degrees of freedom at the element boundaries and only displacement in the domain. The classical and non-classical boundary conditions associated with the gradient beam theory are implemented accurately. The efficiency and accuracy of the element is established through numerical examples on static, free vibration and stability analysis of prismatic and non-prismatic gradient beams.

\section{Gradient elastic beam theory}

In this study, we consider Mindlin's [34] simplified strain gradient micro-elasticity theory with two classical and one nonclassical material constants. The two classical material constants are Lame' constants and the non-classical one is related to intrinsic length $g$. The stress-strain relations for a gradient elastic Euler-Bernoulli beam theory are defined as [37,42]

$$
\tau=E \varepsilon ; \quad \varsigma=g^{2} E \varepsilon^{\prime} ; \quad \varepsilon=-z \frac{\partial^{2} w(x, t)}{\partial x^{2}}
$$

where $\tau, \varsigma$ denote Cauchy and higher order stresses, respectively, $\varepsilon$ is the classical strain and $E$ is the Young's modulus. $z$ is the co-ordinate in thickness direction and $w(x, t)$ is the transverse displacement of the beam. For the above state of stress and strain, the strain energy in bending and due to effect of axial compressive force $P(x)$ for a gradient elastic beam defined over a domain $-L / 2 \leq x \leq L / 2$ can be written as [42]

$$
U=\frac{1}{2} \int_{-L / 2}^{L / 2} E I\left[\left(w^{\prime \prime}\right)^{2}+g^{2}\left(w^{\prime \prime \prime}\right)^{2}\right] d x-\frac{1}{2} \int_{-L / 2}^{L / 2} P\left(w^{\prime}\right)^{2} d x
$$

The work done by the applied load is given by

$$
\bar{W}=-\int_{-L / 2}^{L / 2} q(x) w d x-[V w]_{-L / 2}^{L / 2}+\left[M w^{\prime}\right]_{-L / 2}^{L / 2}+\left[\bar{M} w^{\prime \prime}\right]_{-L / 2}^{L / 2}
$$

The kinetic energy is given as

$$
\bar{K}=\frac{1}{2} \int_{-L / 2}^{L / 2} \rho A \dot{w}^{2} d x
$$




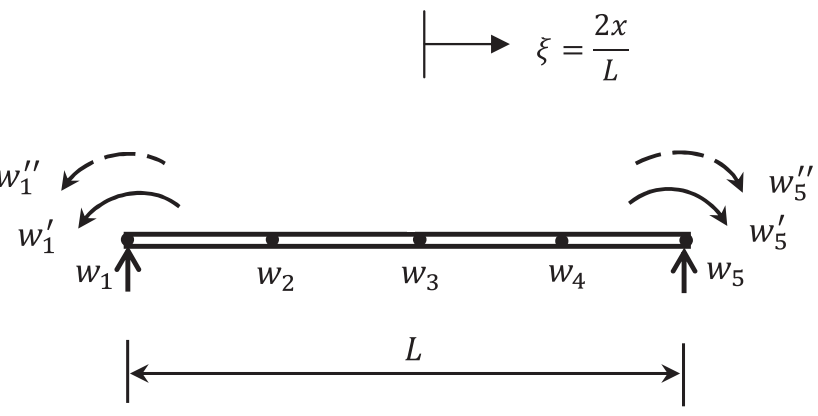

Fig. 1. A five-node quadrature element for a gradient Euler-Bernoulli beam.

where $L, A, I$ and $\rho$ are the length, area, moment of inertia, and density, respectively. $q(x)$ is the transverse distributed load on the beam. $V, M$ and $\bar{M}$ are shear force, bending moment and higher order moment acting on the beam. Over dot indicates differentiation with respect to time.

Substituting Eqs. (2)-(4) in the Hamilton's principle [43]:

$$
\delta \int_{t_{0}}^{t_{1}}(U-\bar{W}-\bar{K}) d t=0
$$

and taking the first variation we obtain

$$
\begin{aligned}
& \int_{t_{0}}^{t_{1}} \int_{-L / 2}^{L / 2} E I\left[w^{\prime \prime} \delta w^{\prime \prime}+g^{2} w^{\prime \prime \prime} \delta w^{\prime \prime \prime}\right] d x d t-\int_{t_{0}}^{t_{1}} \int_{-L / 2}^{L / 2} P w^{\prime} \delta w^{\prime} d x d t+\int_{t_{0}}^{t_{1}} \int_{-L / 2}^{L / 2} q \delta w d x d t- \\
& \int_{t_{0}}^{t_{1}} \int_{-L / 2}^{L / 2} \rho A \dot{w} \delta \dot{w} d x d t+\int_{t_{0}}^{t_{1}}[V \delta w]_{-L / 2}^{L / 2} d t-\int_{t_{0}}^{t_{1}}\left[M \delta w^{\prime}\right]_{-L / 2}^{L / 2} d t-\int_{t_{0}}^{t_{1}}\left[\bar{M} \delta w^{\prime \prime}\right]_{-L / 2}^{L / 2} d t=0
\end{aligned}
$$

Performing the integration-by-parts on Eq. (6), and re-arranging the terms we get

$$
\begin{gathered}
\int_{t_{0}}^{t_{1}} \int_{-L / 2}^{L / 2}\left[E I\left\{w^{i v}-g^{2} w^{v i}\right\}+P w^{\prime \prime}+\rho A \ddot{w}+q\right] \delta w d x d t+\int_{t_{0}}^{t_{1}}\left[\left\{V-\left\{E I\left(w^{\prime \prime \prime}-g^{2} w^{v}\right)+P w^{\prime}\right\}\right\} \delta w\right]_{-L / 2}^{L / 2} d t+ \\
\int_{t_{0}}^{t_{1}}\left[\left\{-M+E I\left(w^{\prime \prime}-g^{2} w^{i v}\right)\right\} \delta w^{\prime}\right]_{-L / 2}^{L / 2} d t+\int_{t_{0}}^{t_{1}}\left[\left\{-\bar{M}+g^{2} E I w^{\prime \prime \prime}\right\} \delta w^{\prime \prime}\right]_{-L / 2}^{L / 2} d t=0
\end{gathered}
$$

The variational equation Eq. (7) requires that each term must be equal to zero, independently. Hence, the governing equilibrium equation for a strain gradient Euler-Bernoulli beam is obtained as

$$
E I\left(w^{i v}-g^{2} w^{v i}\right)+q+P w^{\prime \prime}+\rho A \ddot{w}=0
$$

and the corresponding six boundary conditions are

Classical boundary conditions :

$$
\begin{aligned}
& V=E I\left[w^{\prime \prime \prime}-g^{2} w^{v}\right]+P w^{\prime}=0 \quad \text { or } w=0, \quad \text { at } x=(-L / 2, L / 2) \\
& M=E I\left[w^{\prime \prime}-g^{2} w^{i v}\right]=0 \text { or } w^{\prime}=0, \quad \text { at } x=(-L / 2, L / 2)
\end{aligned}
$$

Non-classical boundary conditions :

$$
\bar{M}=\left[g^{2} E I w^{\prime \prime \prime}\right]=0 \quad \text { or } w^{\prime \prime}=0, \quad \text { at } x=(-L / 2, L / 2)
$$

\section{Quadrature element for a gradient elastic Euler-Bernoulli beam}

A five-node quadrature element for an Euler-Bernoulli gradient beam is shown in Fig. 1. It can be noticed that, each interior node has only displacement $w$ as degrees of freedom and the boundary nodes have 3 degrees of freedom $w, w^{\prime}, w^{\prime \prime}$. The displacement vector now includes the slope and curvature as additional degrees of freedom at the element boundaries given by: $w^{b}=\left\{w_{1}, \ldots, w_{N}, w_{1}^{\prime}, w_{N}^{\prime}, w_{1}^{\prime \prime}, w_{N}^{\prime \prime}\right\}$.

The displacement for a $N$-node quadrature beam element based on Hermite interpolation is assumed as:

$$
w(x, t)=\sum_{j=1}^{N} \phi_{j}(x) w\left(x_{j}, t\right)_{j}+\psi_{1}(x) w^{\prime}\left(x_{1}, t\right)+\psi_{N}(x) w^{\prime}\left(x_{N}, t\right)+\varphi_{1}(x) w^{\prime \prime}\left(x_{1}, t\right)+\varphi_{N}(x) w^{\prime \prime}\left(x_{N}, t\right)=\sum_{j=1}^{N+4} \Gamma_{j}(x) w_{j}^{b}
$$


Expressing in terms of normalized co-ordinate $\xi$ we get

$$
w(\xi, t)=\sum_{j=1}^{N} \phi_{j}(\xi) w\left(\xi_{j}, t\right)+\psi_{1}(\xi) w^{\prime}\left(\xi_{1}, t\right)+\psi_{N}(\xi) w^{\prime}\left(\xi_{N}, t\right)+\varphi_{1}(\xi) w^{\prime \prime}\left(\xi_{1}, t\right)+\varphi_{N}(\xi) w^{\prime \prime}\left(\xi_{N}, t\right)=\sum_{j=1}^{N+4} \Gamma_{j}(\xi) w_{j}^{b}
$$

$\phi, \psi$ and $\varphi$ are Hermite interpolation functions defined as [17]

$$
\begin{aligned}
\varphi_{j}(\xi)= & \frac{1}{2\left(\xi_{j}-\xi_{N-j+1}\right)^{2}} L_{j}(\xi)\left(\xi-\xi_{j}\right)^{2}\left(\xi-\xi_{N-j+1}\right)^{2} \quad(j=1, N) \\
\psi_{j}(\xi)= & \frac{1}{\left(\xi_{j}-\xi_{N-j+1}\right)^{2}} L_{j}(\xi)\left(\xi-\xi_{j}\right)\left(\xi-\xi_{N-j+1}\right)^{2}-\left[2 L_{j}^{\prime}\left(\xi_{j}\right)+\frac{4}{\xi_{j}-\xi_{N-j+1}}\right] \varphi_{j}(\xi) \quad(j=1, N) \\
\phi_{j}(\xi)= & \frac{1}{\left(\xi_{j}-\xi_{N-j+1}\right)^{2}} L_{j}(\xi)\left(\xi-\xi_{N-j+1}\right)^{2}-\left[L_{j}^{\prime}\left(\xi_{j}\right)+\frac{2}{\xi_{j}-\xi_{N-j+1}}\right] \psi_{j}(\xi) \\
& -\left[L_{j}^{\prime \prime}\left(\xi_{j}\right)+\frac{4 L_{j}^{\prime}\left(\xi_{j}\right)}{\xi_{j}-\xi_{N-j+1}}+\frac{2}{\left(\xi_{j}-\xi_{N-j+1}\right)^{2}}\right] \varphi_{j}(\xi) \quad(j=1, N) \\
\phi_{j}(\xi)= & \frac{1}{\left(\xi_{j}-\xi_{1}\right)^{2}\left(\xi_{j}-\xi_{N}\right)^{2}} L_{j}(\xi)\left(\xi-\xi_{1}\right)^{2}\left(\xi-\xi_{N}\right)^{2} \quad(j=2,3, \ldots, N-1)
\end{aligned}
$$

where $L_{j}(\xi)$ is the Lagrange interpolation function in $\xi$ co-ordinate, and $\xi=2 x / L$ with $\xi \in[-1,1]$. The Lagrange interpolation function is defined as $[6,11]$

$$
L_{j}(\xi)=\frac{\beta(\xi)}{\beta\left(\xi_{j}\right)}=\prod_{\substack{k=1 \\ k \neq j}}^{N} \frac{\left(\xi-\xi_{k}\right)}{\left(\xi_{j}-\xi_{k}\right)}
$$

where

$$
\begin{aligned}
& \beta(\xi)=\left(\xi-\xi_{1}\right)\left(\xi-\xi_{2}\right) \cdots\left(\xi-\xi_{j-1}\right)\left(\xi-\xi_{j+1}\right) \cdots\left(\xi-\xi_{N}\right) \\
& \left.\beta\left(\xi_{j}\right)=\left(\xi_{j}-\xi_{1}\right)\left(\xi_{j}-\xi_{2}\right) \cdots\left(\xi_{j}-\xi_{j-1}\right)\left(\xi_{j}-\xi_{j+1}\right) \cdots\right)\left(\xi_{j}-\xi_{N}\right)
\end{aligned}
$$

The $k$ th order derivative of $w(x, t)$ with respect to $x$ is obtained from Eq. (11) as

$$
w^{k}(x, t)=\sum_{j=1}^{N} \phi_{j}^{k}(x) w\left(x_{j}, t\right)+\psi_{1}^{k}(x) w^{\prime}\left(x_{1}, t\right)+\psi_{N}^{k}(x) w^{\prime}\left(x_{N}, t\right)+\varphi_{1}^{k}(x) w^{\prime \prime}\left(x_{1}, t\right)+\varphi_{N}^{k}(x) w^{\prime \prime}\left(x_{N}, t\right)=\sum_{j=1}^{N+4} \Gamma_{j}^{k}(x) w_{j}^{b}
$$

Now, considering the strain energy due to bending given in Eq. (2) and substituting the displacement field given by Eq. (18), we get

$$
U=\frac{1}{2} \int_{-L / 2}^{L / 2}\left\{E I\left[\sum_{i=1}^{N+4} \Gamma_{i}^{\prime \prime}(x) w_{i}^{b}(t)\right]^{2}+g^{2} E I\left[\sum_{i=1}^{N+4} \Gamma_{i}^{\prime \prime \prime}(x) w_{i}^{b}(t)\right]^{2}\right\} d x=\frac{1}{2}\left\{w^{b}\right\}^{T}[K]\left\{w^{b}\right\}
$$

Similarly, the work done by a constant axial compressive force $P$ is given as

$$
\frac{P}{2} \int_{-L / 2}^{L / 2}\left[\sum_{i=1}^{N} \Gamma_{i}^{\prime}(x) w_{i}^{b}(t)\right]^{2} d x=\frac{1}{2}\left\{w^{b}\right\}^{T}[G]\left\{w^{b}\right\}
$$

and the kinetic energy given in Eq. (4) is expressed as

$$
\frac{1}{2} \int_{-L / 2}^{L / 2} \rho A\left[\sum_{i=1}^{N} \Gamma_{i}(x) \dot{w}_{i}^{b}(t)\right]^{2} d x=\frac{1}{2}\left\{\dot{w}^{b}\right\}^{T}[m]\left\{\dot{w}^{b}\right\}
$$

where $K$ is the elastic stiffness matrix, $G$ is the geometric stiffness matrix, $m$ is the mass matrix defined as below

$$
\begin{aligned}
K_{i j} & =E I \int_{-L / 2}^{L / 2}\left\{\Gamma_{i}^{\prime \prime}(x) \Gamma_{j}^{\prime \prime}(x)+g^{2} \Gamma_{i}^{\prime \prime \prime}(x) \Gamma_{j}^{\prime \prime \prime}(x)\right\} d x=E I \int_{-1}^{1}\left\{\frac{16}{L^{4}} \Gamma_{i}^{\prime \prime}(\xi) \Gamma_{j}^{\prime \prime}(\xi)+g^{2} \frac{64}{L^{6}} \Gamma_{i}^{\prime \prime \prime}(\xi) \Gamma_{j}^{\prime \prime \prime}(\xi)\right\}|\bar{J}(\xi)| d \xi \\
& =\frac{8 E I}{L^{3}} \sum_{k=1}^{N} H_{k} \Gamma_{i}^{\prime \prime}\left(\xi_{k}\right) \Gamma_{j}^{\prime \prime}\left(\xi_{k}\right)+g^{2} \frac{32 E I}{L^{5}} \sum_{k=1}^{N} H_{k} \Gamma_{i}^{\prime \prime \prime}\left(\xi_{k}\right) \Gamma_{j}^{\prime \prime \prime}\left(\xi_{k}\right) \quad(i, j=1,2, \ldots, N, N+1, \ldots, N+4)
\end{aligned}
$$




$$
\begin{gathered}
G_{i j}=P \int_{-L / 2}^{L / 2} \Gamma_{i}^{\prime}(x) \Gamma_{j}^{\prime}(x) d x=\frac{4 P}{L^{2}} \int_{-1}^{1} \Gamma_{i}^{\prime}(\xi) \Gamma_{j}^{\prime}(\xi)|\bar{J}(\xi)| d \xi=\frac{2 P}{L} \sum_{k=1}^{N} H_{k} \Gamma_{i}^{\prime}\left(\xi_{k}\right) \Gamma_{j}^{\prime}\left(\xi_{k}\right) \quad(i, j=1,2, \ldots, N) \\
m_{i j}=\int_{-L / 2}^{L / 2} \rho A \Gamma_{i}(x) \Gamma_{j}(x) d x=\rho A \int_{-1}^{1} \Gamma_{i}(\xi) \Gamma_{j}(\xi)|\bar{J}(\xi)| d \xi=\frac{\rho A L}{2} \sum_{k=1}^{N} H_{k} \Gamma_{i}\left(\xi_{k}\right) \Gamma_{j}\left(\xi_{k}\right) \\
=\frac{\rho A L}{2} \sum_{k=1}^{N} H_{k} \delta_{k i} \delta_{k j}=\frac{\rho A L}{2} H_{i} \delta_{i j} \quad(i, j=1,2, \ldots, N)
\end{gathered}
$$

and finally the load vector due to transverse distributed load is given by

$$
f_{i}=\int_{-L / 2}^{L / 2} q(x) \Gamma_{i}(x) d x=\frac{L}{2} \int_{-1}^{1} q(\xi) \Gamma_{i}(\xi) d x=\frac{L}{2} H_{i} q\left(\xi_{i}\right) \quad(i=1,2, \ldots, N)
$$

where $\xi_{i}$ and $H_{i}$ are GLL quadrature co-ordinates and weights, respectively, $|\bar{J}(\xi)|=L / 2$ is the Jacobian, $\delta_{i j}$ is the Dirac-delta function, with a value of 1 if $(i=j)$ and 0 if $(i \neq j)$. It is to be noted that in the geometric stiffness matrix Eq. (23), the mass matrix Eq. (24) and the load vector Eq. (25) the contribution from deflection degrees of freedom are only considered as the contribution from derivative degrees of freedom are identically zero.

Combining Eqs. (22)-(25), the system of equations can be partitioned based on the boundary and domain degrees of freedom as [6]

$$
\left[\begin{array}{cc}
K_{b b} & K_{b d} \\
K_{d b} & K_{d d}
\end{array}\right]\left\{\begin{array}{c}
\Delta_{b} \\
\Delta_{d}
\end{array}\right\}=\left\{\begin{array}{l}
\bar{f}_{b} \\
\bar{f}_{d}
\end{array}\right\}+\left[\begin{array}{cc}
I & 0 \\
0 & \omega^{2} m_{d d}
\end{array}\right]\left\{\begin{array}{c}
0 \\
\Delta_{d}
\end{array}\right\}+P\left[\begin{array}{cc}
0 & 0 \\
G_{d b} & G_{d d}
\end{array}\right]\left\{\begin{array}{c}
\Delta_{b} \\
\Delta_{d}
\end{array}\right\}
$$

where the subscript ' $b$ ' and ' $d$ ' corresponds to boundary and domain of the element. The vector $\Delta_{b}$ contains the boundary related non-zero slope and curvature degrees of freedom. Similarly, the vector $\Delta_{d}$ includes all the non-zero displacement degrees of freedom of the beam. The vectors $\bar{f}_{b}$ and $\bar{f}_{d}$ represents boundary and domain forces. I is the identity matrix. For bending analysis $m_{d d}=P=0$, for free vibration analysis $\bar{f}_{b}=\bar{f}_{d}=P=0$, and $\bar{f}_{b}=\bar{f}_{d}=m_{d d}=0$ for stability analysis. The solution of the Eq. (26) after applying the boundary conditions leads to unknown displacements, frequencies and buckling load.

\section{Numerical results and discussion}

The convergence and accuracy of the proposed quadrature beam element is assessed for static, free vibration and stability analysis of gradient Euler-Bernoulli beams using exact solutions. The generality and applicability of the proposed element is verified for gradient beams with prismatic and non-prismatic cross section under different boundary and loading conditions. Beams with discontinuity in geometry or loading are modelled using two elements and the assembly procedure is similar to the conventional finite element method. The numerical data used for the analysis of beams in consistent units is as follows: Length $L=1$, Young's modulus $E=3 \times 10^{6}$, Poisson's ratio $v=0.3$, density $\rho=1$ and transverse load $q=1$.

The classical and non-classical boundary conditions used in the present analysis for different end support are:

Simply supported:

classical: $w=M=0, \quad$ non-classical $: w^{\prime \prime}=0$ at $x=\left(-\frac{L}{2}, \frac{L}{2}\right)$

Clamped:

classical: $\quad w=w^{\prime}=0, \quad$ non-classical $: w^{\prime \prime}=0$ at $x=\left(-\frac{L}{2}, \frac{L}{2}\right)$

Free-free:

classical: $Q=M=0, \quad$ non-classical $: \bar{M}=0 \quad$ at $x=\left(-\frac{L}{2}, \frac{L}{2}\right)$

Cantilever:

classical: $w=w^{\prime}=0 \quad$ at $\quad x=-\frac{L}{2}, Q=M=0$ at $\quad x=\frac{L}{2}$

non-classical: $w^{\prime \prime}=0$ at $x=-\frac{L}{2}, \quad \bar{M}=0 \quad$ at $\quad x=\frac{L}{2}$

Propped cantilever:

classical : $w=w^{\prime}=0 \quad$ at $x=-\frac{L}{2}, w=M=0 \quad$ at $\quad x=\frac{L}{2}$

non-classical : $w^{\prime \prime}=0 \quad$ at $x=-\frac{L}{2}, w^{\prime \prime}=0 \quad$ at $\quad x=\frac{L}{2}$

Pinned-guided:

classical: $w=M=0 \quad$ at $x=-\frac{L}{2}, V=w^{\prime}=0 \quad$ at $\quad x=\frac{L}{2}$

non-classical: $w^{\prime \prime}=0 \quad$ at $x=-\frac{L}{2}, \bar{M}=0 \quad$ at $\quad x=\frac{L}{2}$

For a beam with single element mesh, the size of the displacement vector $\Delta_{d}$ defined in Eq. (26) for simply supported, clamped and propped cantilever boundary conditions remains as $(N-2)$ and for free-free, cantilever and pinned-guided beam the size is $N,(N-1)$ and $(N-1)$, respectively. With multiple elements discretization ' $\bar{N}_{\text {elm }}$ ' the size of the displacement 
Table 1

Comparison of deflection, slope for a simply supported beam under a udl.

\begin{tabular}{|c|c|c|c|c|c|c|}
\hline \multirow[t]{2}{*}{$N$} & \multicolumn{2}{|c|}{$g / L=0.05$} & \multicolumn{2}{|c|}{$g / L=0.1$} & \multicolumn{2}{|c|}{$g / L=0.2$} \\
\hline & $w_{(x=0)}$ & $w_{(x=-L / 2)}^{\prime}$ & $w_{(x=0)}$ & $w_{(x=-L / 2)}^{\prime}$ & $w_{(x=0)}$ & $w_{(x=-L / 2)}^{\prime}$ \\
\hline 5 & 1.3378 & 0.1664 & 1.1978 & 0.1543 & 0.9332 & 0.1194 \\
\hline 7 & 1.2715 & 0.1622 & 1.1868 & 0.1506 & 0.9355 & 0.1182 \\
\hline 9 & 1.2715 & 0.1622 & 1.1870 & 0.1507 & 0.9360 & 0.1182 \\
\hline 11 & 1.2714 & 0.1622 & 1.1869 & 0.1507 & 0.9360 & 0.1182 \\
\hline 13 & 1.2714 & 0.1622 & 1.1869 & 0.1507 & 0.9360 & 0.1182 \\
\hline 15 & 1.2714 & 0.1622 & 1.1869 & 0.1507 & 0.9360 & 0.1182 \\
\hline Exact* & 1.2714 & 0.1622 & 1.1869 & 0.1507 & 0.9360 & 0.1182 \\
\hline
\end{tabular}

* See Appendix.

Table 2

Comparison of deflection, slope and curvature at the tip for a cantilever beam under a udl.

\begin{tabular}{|c|c|c|c|c|c|c|}
\hline \multirow[t]{2}{*}{$N$} & \multicolumn{3}{|c|}{$g / L=0.05$} & \multicolumn{3}{|l|}{$g / L=0.1$} \\
\hline & $w_{(x=L / 2)}$ & $w_{(x=L / 2)}^{\prime}$ & $w_{(x=L / 2)}^{\prime \prime}$ & $w_{(x=L / 2)}$ & $w_{(x=L / 2)}^{\prime}$ & $w_{(x=L / 2)}^{\prime \prime}$ \\
\hline 5 & 9.4324 & 0.5439 & 0.0084 & 8.3466 & 0.5008 & 0.0305 \\
\hline 7 & 10.1928 & 0.5744 & 0.0233 & 8.4305 & 0.5036 & 0.0285 \\
\hline 9 & 10.2397 & 0.5762 & 0.0042 & 8.4106 & 0.5027 & 0.0395 \\
\hline 11 & 10.2385 & 0.5762 & 0.0095 & 8.4099 & 0.5027 & 0.0398 \\
\hline 13 & 10.2381 & 0.5762 & 0.0100 & 8.4099 & 0.5027 & 0.0398 \\
\hline 15 & 10.2381 & 0.5762 & 0.0100 & 8.4099 & 0.5027 & 0.0398 \\
\hline Exact [37] & 10.2381 & 0.5762 & 0.0100 & 8.4099 & 0.5027 & 0.0398 \\
\hline
\end{tabular}

vector $\Delta_{d}$ for simply supported, clamped and propped cantilever boundary conditions will be $\left(\hat{N}_{e l m} \times(N-1)-1\right)$ and for free-free, cantilever and pinned-guided beam it is $\left(\hat{N}_{e l m} \times(N-1)+1\right),\left(\hat{N}_{e l m} \times(N-1)\right)$ and $\left(\hat{N}_{e l m} \times(N-1)\right)$, respectively. However, the size of the $\Delta_{b}$ vector depends upon the number of non-zero slope and curvature degrees of freedom at the element and inter-element boundaries. The non-classical boundary conditions employed for simply supported gradient beam are $w^{\prime \prime}=0$ at $x=\left(-\frac{L}{2}, \frac{L}{2}\right)$, the equations related to curvature degrees of freedom are eliminated and the size of $\Delta_{b}$ is 2 for single element mesh and $2 \hat{N}_{e l m}$ for multiple elements. For the gradient cantilever beam the non-classical boundary conditions used are $w^{\prime \prime}=0$ at $x=-\frac{L}{2}$ and $\bar{M}=0$ at $x=\frac{L}{2}$. The equation related to curvature degrees of freedom at $x=-\frac{L}{2}$ is eliminated and the equation related to higher order moment at $x=\frac{L}{2}$ is retained and the size of $\Delta_{b}=2$ for single beam element and $2 \hat{N}_{\text {elm }}$ for multiple elements. In the case of clamped beam the non-classical boundary conditions read $w^{\prime \prime}=0$ at $x=\left(-\frac{L}{2}, \frac{L}{2}\right)$ and the $\Delta_{b}$ is zero and $2\left(\hat{N}_{e l m}-1\right)$ for single and multiple element mesh, respectively. Similarly, for propped cantilever beam we get 1 and $\left(2 \hat{N}_{e l m}-1\right)$, as $w^{\prime \prime}=0$ at $x=\left(-\frac{L}{2}, \frac{L}{2}\right)$. For a free-free beam the size of $\Delta_{b}$ is 4 and $2\left(\hat{N}_{e l m}+1\right)$ due to $\bar{M}=0$ at $x=\left(-\frac{L}{2}, \frac{L}{2}\right)$ and finally, for a pinned-guided beam the size of $\Delta_{b}$ is 3 and $2\left(\hat{N}_{e l m}+1\right)$.

\subsection{Static analysis of gradient elastic beams using quadrature element}

Here we consider two class of problems, beams subjected to uniformly distributed load (udl) and beams under concentrated load. The prismatic beams under uniformly distributed load are modelled using single quadrature element and the results are compared with analytical solutions given by Papargyri et al. [37] and those derived in Appendix. Papargyri et al. [37] presented results only for cantilever beam and for other boundary conditions analytical solutions are derived in the Appendix. The beams with geometry or load discontinuity are discretized using two quadrature elements and the comparisons are made with Pegios et al. [41] results. Pegios et al. [41] derived the stiffness matrix analytically by assuming the exact solution to the governing equation as displacement function and taking $\left\{w, w^{\prime}, w^{\prime \prime}\right\}^{\mathrm{T}}$ as degrees of freedom at each end of the beam. As a result, the stiffness matrix and the structural behaviour obtained are exact. To solve the examples with discontinuity in loading and geometry they have used exact stiffness matrix for each segment of the beam and employed the finite element (FE) framework for assembly. As the stiffness matrix is exact, the solutions obtained using one element for prismatic beams and two elements mesh for examples chosen in this study are exact. Hence, in the present analysis the results given in Pegios et al. [41] are taken as exact and are designated as FE-Exact for results comparison.

\subsubsection{Gradient elastic beams under uniformly distributed load}

Three different beam support conditions are considered in this study, simply supported, cantilever and pinned-guided. The results reported here for beams with uniformly distributed load are non-dimensional as, deflection $: \bar{w}=100 E I w / q L^{4}$ and curvature $: \bar{w}^{\prime \prime}=w^{\prime \prime} L$.

In Table 1, convergence of non-dimensional deflection and slope for a simply supported beam are given for $g / L=0.05,0.1$ and 0.2. The deflection is evaluated at center of the beam $x=0$ and slope are computed at the left support $x=-L / 2$. Good 
Table 3

Comparison of deflection and slope for a pinned-guided beam under a udl.

\begin{tabular}{|c|c|c|c|c|c|c|}
\hline \multirow[t]{2}{*}{$N$} & \multicolumn{2}{|c|}{$g / L=0.05$} & \multicolumn{2}{|l|}{$g / L=0.1$} & \multicolumn{2}{|l|}{$g / L=0.2$} \\
\hline & $w_{(x=0)}$ & $w_{(x=-L / 2)}^{\prime}$ & $w_{(x=0)}$ & $w_{(x=-L / 2)}^{\prime}$ & $w_{(x=0)}$ & $w_{(x=-L / 2)}^{\prime}$ \\
\hline 5 & 20.7130 & 1.3283 & 20.3525 & 1.3014 & 19.0016 & 1.2075 \\
\hline 7 & 20.7089 & 1.3238 & 20.3433 & 1.2973 & 18.9911 & 1.2053 \\
\hline 9 & 20.7089 & 1.3238 & 20.3433 & 1.2973 & 18.9912 & 1.2053 \\
\hline 11 & 20.7089 & 1.3238 & 20.3433 & 1.2973 & 18.9912 & 1.2053 \\
\hline 13 & 20.7089 & 1.3238 & 20.3433 & 1.2973 & 18.9912 & 1.2053 \\
\hline 15 & 20.7089 & 1.3238 & 20.3433 & 1.2973 & 18.9912 & 1.2053 \\
\hline Exact* & 20.7089 & 1.3238 & 20.3433 & 1.2973 & 18.9912 & 1.2053 \\
\hline
\end{tabular}

* See Appendix.

Table 4

Comparison of deflection along the length for a pinned-guided beam under a udl.

\begin{tabular}{|c|c|c|c|c|c|c|}
\hline \multirow[t]{2}{*}{$\xi=2 x / L$} & \multicolumn{2}{|c|}{$g / L=0.05$} & \multicolumn{2}{|l|}{$g / L=0.1$} & \multicolumn{2}{|c|}{$g / L=0.2$} \\
\hline & Present & Exact* & Present & Exact* & Present & Exact* \\
\hline-1. & 0 & 0 & 0 & & 00 & 0.0000 \\
\hline-0.8302 & 2.7998 & 2.7998 & 2.7440 & 10 & 498 & 2.5498 \\
\hline-0.4688 & 8.5041 & 50 & .3401 & 8.3 & 7.7576 & 7.7576 \\
\hline 0.0000 & 14.7506 & 14.7506 & 14.4786 & 14.4786 & 13.4903 & 13.4903 \\
\hline 0.4688 & 18.9752 & 18.9752 & 18.6361 & 18.6361 & 17.3877 & 17.3877 \\
\hline 0.8302 & 20.5299 & 20.5299 & 20.1669 & 20.1669 & 18.8254 & 18.8254 \\
\hline 1.0000 & 20.7089 & 20.7089 & 20.3433 & 20.3433 & 18.9912 & 18.9912 \\
\hline
\end{tabular}

* See Appendix.

Table 5

Comparison of maximum non-dimensional deflection for a simply supported beam under a udl for a sequence of $g / L$ values.

\begin{tabular}{llllll}
\hline \multirow{2}{*}{$g / L$} & $N$ & & & & \\
\cline { 2 - 6 } & 5 & 7 & 9 & 11 & Exact* \\
\hline 0.01 & 3.0429 & 1.3008 & 1.3008 & 1.3008 & 1.3008 \\
0.03 & 1.4823 & 1.2909 & 1.2909 & 1.2909 & 1.2909 \\
0.05 & 1.3378 & 1.2715 & 1.2714 & 1.2714 & 1.2714 \\
0.07 & 1.2741 & 1.2432 & 1.2432 & 1.2432 & 1.2432 \\
0.09 & 1.2228 & 1.2072 & 1.2074 & 1.2074 & 1.2074 \\
0.10 & 1.1978 & 1.1868 & 1.1870 & 1.1869 & 1.1869 \\
0.11 & 1.1725 & 1.1649 & 1.1652 & 1.1652 & 1.1652 \\
0.13 & 1.1206 & 1.1179 & 1.1182 & 1.1182 & 1.1182 \\
0.15 & 1.0674 & 1.0675 & 1.0679 & 1.0678 & 1.0678 \\
0.17 & 1.0134 & 1.0151 & 1.0156 & 1.0155 & 1.0155 \\
0.19 & 0.9597 & 0.9620 & 0.9625 & 0.9625 & 0.9625 \\
0.20 & 0.9332 & 0.9355 & 0.9360 & 0.9360 & 0.9360 \\
\hline
\end{tabular}

* See Appendix.

convergence is exhibited by the present element, with deflection and slope approaching to exact values with 11 nodes or grid points. In Table 2, the convergence behaviour of non-dimensional deflection, slope and curvature are presented for a cantilever beam. The deflection, slope and curvature are obtained at the right support $x=L / 2$. Similar convergence is demonstrated by the present element for both classical and non-classical quantities for $g / L=0.05$ and 0.1 . Excellent agreement with the exact solution is seen with 9 nodes or grid points and converged solutions are obtained with 13 nodes or grid points.

In Table 3, convergence behaviour of non-dimensional deflection and slope for a pinned-guided support are presented for $g / L=0.05,0.1$ and 0.2 . The deflection is compared at $x=0$ and slope at $x=-L / 2$ with the exact solutions. Very good convergence trend is displayed by the present element for both deflection and slope with solution approaching to the exact values with 7 nodes. This consistency is noticed for all the $g / L$ values considered.

In Table 4, non-dimensional deflection is compared along the length of the beam for a pinned-guided support. The deflection is computed using 7 nodes and excellent agreement is exhibited with the exact solutions for the entire length of the beam for all the $g / L$ values considered.

In Table 5, the convergence of maximum non-dimensional deflection for a simply supported beam is shown for a sequence of $g / L$ values. Accurate results are obtained using 9 nodes and convergence is attained with 11 nodes for all the $g / L$ values. 

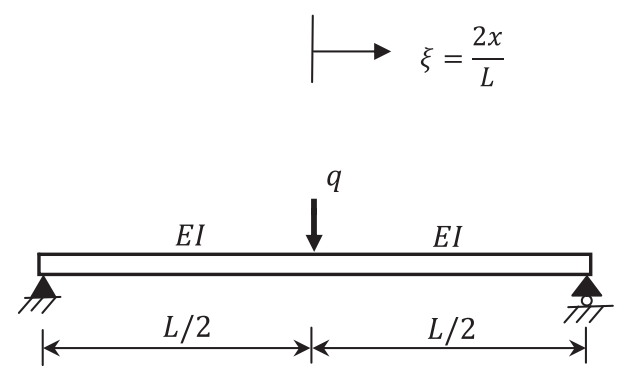

Fig. 2. A simply supported gradient beam under a middle point load.
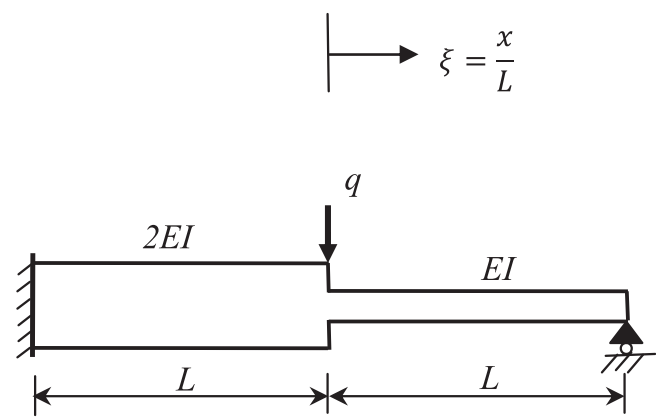

Fig. 3. A stepped propped cantilever beam under a middle point load.

Table 6

Comparison of non-dimensional deflection and slope for a simply supported beam under a middle point load.

\begin{tabular}{|c|c|c|c|c|c|c|}
\hline \multirow{2}{*}{$\begin{array}{l}N \\
\text { (per element) }\end{array}$} & \multicolumn{2}{|c|}{$g / L=0.05$} & \multicolumn{2}{|c|}{$g / L=0.1$} & \multicolumn{2}{|c|}{$g / L=0.2$} \\
\hline & $w_{(x=0)}$ & $w_{(x=-L / 2)}^{\prime}$ & $w_{(x=0)}$ & $w_{(x=-L / 2)}^{\prime}$ & $w_{(x=0)}$ & $w_{(x=-L / 2)}^{\prime}$ \\
\hline 5 & 2.0271 & 0.2453 & 1.8848 & 0.2309 & 1.4801 & 0.1835 \\
\hline 7 & 2.0271 & 0.2450 & 1.8833 & 0.2303 & 1.4780 & 0.1830 \\
\hline 9 & 2.0271 & 0.2450 & 1.8833 & 0.2303 & 1.4780 & 0.1830 \\
\hline 11 & 2.0271 & 0.2450 & 1.8833 & 0.2303 & 1.4780 & 0.1830 \\
\hline 13 & 2.0271 & 0.2450 & 1.8833 & 0.2303 & 1.4780 & 0.1830 \\
\hline 15 & 2.0271 & 0.2450 & 1.8833 & 0.2303 & 1.4780 & 0.1830 \\
\hline FE-Exact [41] & 2.0271 & 0.2450 & 1.8833 & 0.2303 & 1.4780 & 0.1830 \\
\hline
\end{tabular}

From the above observations it is concluded that, a single quadrature beam element with reasonable number of nodes can be efficiently applied to study the static behaviour of gradient elastic Euler-Bernoulli beam under udl for different choice of intrinsic length and boundary condition.

\subsubsection{Gradient elastic beams with concentrated load}

To establish the capability of the proposed element for beams with concentrated load two examples are considered, a simply supported and a stepped propped cantilever beam with middle point load as shown in Figs. 2 and 3 , respectively. Two quadrature elements are used to model the beam. Comparison is made with the exact solutions given by Pegios et al. [41] (FE-Exact) which are obtained using two finite elements with 6 degrees of freedom $\left\{w_{1}, w_{1}^{\prime}, w_{1}^{\prime \prime}, w_{2}, w_{2}^{\prime}, w_{2}^{\prime \prime}\right\}^{T}$ per element. In Table 6, convergence of deflection and slope for a simply supported beam with a middle concentrate load is given for $g / L=0.05,0.1$ and 0.2 . With 7 grid points both deflection and slope converge to exact values for all the three $g / L$ values. Similar convergence trend is noticed in Table 7 , for a stepped propped cantilever beam. Accurate solutions are obtained using 7 nodes or grid points and converged results using 9 nodes or grid points.

From the above tabulated results it can be concluded that the present element can be efficiently applied for static analysis of strain gradient Euler-Bernoulli beams with geometry and load discontinuity.

\subsection{Free vibration analysis of gradient beams using quadrature element}

All the frequencies reported here are non-dimensional as $\bar{\omega}=\omega L^{2} \sqrt{\rho A / E I}$. The frequencies obtained using the quadrature element are compared with analytical solutions presented in Papargyri et al. [38] and those derived in Appendix. Papargyri 
Table 7

Comparison of deflection and slope for a stepped propped cantilever beam under a middle point load.

\begin{tabular}{|c|c|c|c|c|c|c|}
\hline \multirow[t]{2}{*}{$N$} & \multicolumn{2}{|c|}{$g / L=0.05$} & \multicolumn{2}{|c|}{$g / L=0.1$} & \multicolumn{2}{|c|}{$g / L=0.2$} \\
\hline & $w_{(x=0)}$ & $w_{(x=-L)}^{\prime}$ & $w_{(x=0)}$ & $w_{(x=-L)}^{\prime}$ & $w_{(x=0)}$ & $w_{(x=-L)}^{\prime}$ \\
\hline 5 & 0.5425 & 0.3488 & 0.4898 & 0.3303 & 0.3600 & 0.2797 \\
\hline 7 & 0.5624 & 0.3579 & 0.4887 & 0.3294 & 0.3514 & 0.2744 \\
\hline 9 & 0.5636 & 0.3584 & 0.4879 & 0.3290 & 0.3513 & 0.2743 \\
\hline 11 & 0.5635 & 0.3583 & 0.4879 & 0.3290 & 0.3513 & 0.2743 \\
\hline 13 & 0.5635 & 0.3583 & 0.4879 & 0.3290 & 0.3513 & 0.2743 \\
\hline 15 & 0.5635 & 0.3583 & 0.4879 & 0.3290 & 0.3513 & 0.2743 \\
\hline FE-Exact [41] & 0.5635 & 0.3583 & 0.4879 & 0.3290 & 0.3513 & 0.2743 \\
\hline
\end{tabular}

Table 8

Comparison of first six frequencies for a simply supported gradient beam.

\begin{tabular}{llllllll}
\hline$g / L$ & $N$ & $\bar{\omega}_{1}$ & $\bar{\omega}_{2}$ & $\bar{\omega}_{3}$ & $\bar{\omega}_{4}$ & $\bar{\omega}_{5}$ & $\bar{\omega}_{6}$ \\
\hline & 5 & 10.2771 & 48.7221 & 91.5630 & - & - & - \\
& 7 & 10.3450 & 46.6455 & 114.8632 & 304.8997 & 337.6992 & - \\
& 9 & 10.3452 & 46.6244 & 121.8582 & 257.6350 & 299.9480 & 1170.4848 \\
& 11 & 10.3452 & 46.6244 & 122.0583 & 253.6890 & 403.7814 & 620.7953 \\
& 13 & 10.3452 & 46.6244 & 122.0601 & 253.6051 & 457.0636 & 678.4906 \\
& 15 & 10.3452 & 46.6244 & 122.0601 & 253.6045 & 459.4206 & 758.2578 \\
& 17 & 10.3452 & 46.6244 & 122.0601 & 253.6045 & 459.4535 & 758.1492 \\
& 19 & 10.3452 & 46.6244 & 122.0601 & 253.6045 & 459.4537 & 758.1477 \\
& 21 & 10.3452 & 46.6244 & 122.0601 & 253.6045 & 459.4537 & 758.1477 \\
& Analytical [38] & 10.3452 & 46.6244 & 122.0601 & 253.6045 & 459.4537 & 758.1477 \\
\hline & 5 & 11.6511 & 67.8787 & 92.6048 & - & & - \\
& 7 & 11.6561 & 66.4433 & 137.6852 & 415.5394 & 589.1990 & - \\
& 9 & 11.6561 & 63.4012 & 185.7633 & 315.6633 & 434.8071 & 211.1195 \\
& 11 & 11.6561 & 63.4011 & 189.5022 & 427.3027 & 459.2190 & 978.7724 \\
& 13 & 11.6561 & 63.4011 & 189.5368 & 427.1438 & 670.3214 & 840.8526 \\
& 15 & 11.6561 & 63.4011 & 189.5369 & 427.1425 & 812.4993 & 936.4399 \\
& 17 & 11.6561 & 63.4011 & 189.5369 & 427.1425 & 813.4755 & 1226.7108 \\
& 19 & 11.6561 & 63.4011 & 189.5369 & 427.1425 & 813.4795 & 138.5794 \\
& 21 & 11.6561 & 63.4011 & 189.5369 & 427.1425 & 813.4795 & 138.5794 \\
& Analytical [38] & 11.6561 & 63.4011 & 189.5369 & 427.1425 & 813.4795 & 138.5794 \\
\hline
\end{tabular}

et al. [38] gave solutions only for simply supported beam and the frequencies for other boundary conditions used in this study are derived by following the approach given in [44] and the associated frequency equations are presented in Appendix. In this study, single quadrature element is used to generate the results and the rotary inertia related to slope and curvature degrees of freedom is neglected. In Table 8, convergence of first six frequencies for a simply supported beam obtained using the present element for $g / L=0.1,0.5$ are presented and compared with analytical values [38]. Good agreement with the analytical frequencies is seen, with first three frequencies converging to analytical values using $N=13$ nodes. The higher frequencies converge to analytical results with $N=19$ nodes for the $g / L$ values chosen. Similar trend is noticed inTables $9-11$, for cantilever, propped cantilever and free-free beams, respectively. For the free-free beam, the frequencies compared are related to elastic modes and the rigid mode frequencies are not reported here, which are zeros. It is to be noted that, the present elements is efficient in capturing the rigid body modes associated with the generalized degrees of freedom.

Table 12, shows the convergence behaviour of first two frequencies for a simply supported beam obtained using the proposed element for sequence of $g / L$ values. Good agreement with the analytical frequencies is seen for all the $g / L$ values with the frequencies converging to analytical values using $N=9$ nodes.

Hence, the quadrature element can be effectively applied to study the free vibration behaviour of gradient elastic beams with reasonable number of nodes to produce accurate solutions even for higher frequencies for different the boundary conditions and length scale parameters.

\subsection{Stability analysis of gradient elastic beams using quadrature element}

In this section, the applicability of the proposed element for stability analysis of prismatic and non-prismatic gradient Euler-Bernoulli beams will be verified. Comparison is made with the analytical results available in literature and those derived in Appendix for different boundary conditions and $g / L$ values. All the results reported here are obtained using single beam element and non-dimensional as $\bar{P}_{c r}=P_{c r} L^{2} / E I$. For simply supported beam results are compared with Papargyri et al. [37] and Pegios et al. [41] (FE-Exact), for cantilever beam with Papargyri et al. [40] and for remaining boundary conditions with those derived in the Appendix. In Table 13, convergence of critical buckling load for a simply supported prismatic 
Table 9

Comparison of first six frequencies for a cantilever gradient beam.

\begin{tabular}{llllllll}
\hline$g / L$ & $N$ & $\bar{\omega}_{1}$ & $\bar{\omega}_{2}$ & $\bar{\omega}_{3}$ & $\bar{\omega}_{4}$ & $\bar{\omega}_{5}$ & $\bar{\omega}_{6}$ \\
\hline \multirow{4}{*}{0.1} & 5 & 4.3231 & 29.0912 & 101.5130 & 243.6011 & - & - \\
& 7 & 4.3018 & 28.3533 & 88.3366 & 152.5461 & 440.9457 & 1816.5639 \\
& 9 & 4.3073 & 28.4537 & 87.7727 & 191.3261 & 317.8770 & 446.4752 \\
& 11 & 4.3074 & 28.4554 & 87.8027 & 194.4421 & 360.9022 & 463.2194 \\
& 15 & 4.3074 & 28.4554 & 87.8029 & 194.5265 & 365.4698 & 592.0424 \\
& 17 & 4.3074 & 28.4554 & 87.8029 & 194.5273 & 365.5262 & 618.5239 \\
& 19 & 4.3074 & 28.4554 & 87.8029 & 194.5273 & 365.5268 & 618.9979 \\
& 21 & 4.3074 & 28.4554 & 87.8029 & 194.5273 & 365.5268 & 619.0030 \\
& Analytical ${ }^{*}$ & 4.3074 & 28.4554 & 87.8029 & 194.5273 & 365.5268 & 619.0030 \\
& & & & & & & \\
& 5 & 5.1372 & 32.3504 & 110.9295 & 464.2787 & - & \\
& 7 & 5.2181 & 36.8711 & 123.5796 & 166.7896 & 601.7111 & 3614.9546 \\
& 9 & 5.2198 & 36.8772 & 125.2653 & 262.8536 & 372.4766 & 763.5845 \\
& 11 & 5.2198 & 36.8773 & 125.4356 & 304.5895 & 460.0053 & 647.2694 \\
& 13 & 5.2198 & 36.8773 & 125.4358 & 305.3059 & 610.7455 & 683.4273 \\
& 15 & 5.2198 & 36.8773 & 125.4358 & 305.3126 & 614.0537 & 932.6251 \\
& 17 & 5.2198 & 36.8773 & 125.4358 & 305.3126 & 614.0671 & 1089.4434 \\
& 19 & 5.2198 & 36.8773 & 125.4358 & 305.3126 & 614.0672 & 1089.5532 \\
& 21 & 5.2198 & 36.8773 & 125.4358 & 305.3126 & 614.0672 & 1089.5536 \\
& Analytical ${ }^{*}$ & 5.2198 & 36.8773 & 125.4358 & 305.3126 & 614.0672 & 1089.5536 \\
\hline
\end{tabular}

* See Appendix.

Table 10

Comparison of first six frequencies for a propped cantilever gradient beam.

\begin{tabular}{llllllll}
\hline$g / L$ & $N$ & $\bar{\omega}_{1}$ & $\bar{\omega}_{2}$ & $\bar{\omega}_{3}$ & $\bar{\omega}_{4}$ & $\bar{\omega}_{5}$ & $\bar{\omega}_{6}$ \\
\hline & 5 & 20.5259 & 72.7864 & 242.6638 & - & - & - \\
& 7 & 19.9364 & 72.4394 & 148.3416 & 320.8409 & 1822.1783 & - \\
& 9 & 19.9913 & 72.3983 & 172.0579 & 290.7313 & 441.2457 & 1307.7343 \\
& 11 & 19.9926 & 72.4152 & 172.7926 & 334.6391 & 463.2152 & 708.5141 \\
& 13 & 19.9926 & 72.4153 & 172.8227 & 338.6274 & 574.0068 & 691.7580 \\
& 15 & 19.9926 & 72.4153 & 172.8229 & 338.6909 & 588.0822 & 885.3525 \\
& 17 & 19.9926 & 72.4153 & 172.8229 & 338.6915 & 588.3014 & 939.7318 \\
& 19 & 19.9926 & 72.4153 & 172.8229 & 338.6915 & 588.3037 & 940.1743 \\
& 21 & 19.9926 & 72.4153 & 172.8229 & 338.6915 & 588.3037 & 940.1791 \\
& Analytical* & 19.9926 & 72.4153 & 172.8229 & 338.6915 & 588.3037 & 940.1792 \\
\hline & 5 & 25.7751 & 81.7863 & 464.9064 & - & & - \\
& 7 & 27.2146 & 109.1067 & 166.8390 & 510.3642 & 3628.9640 & - \\
& 9 & 27.2328 & 110.9694 & 260.0398 & 344.5498 & 756.9604 & 2429.7140 \\
& 11 & 27.2329 & 110.5674 & 287.6102 & 457.4952 & 631.1660 & 1242.1337 \\
& 13 & 27.2329 & 111.0570 & 288.0483 & 592.5704 & 682.9530 & 1096.9887 \\
& 15 & 27.2329 & 111.0570 & 288.0516 & 595.2458 & 932.5588 & 1073.8457 \\
& 17 & 27.2329 & 111.0570 & 288.0516 & 595.2576 & 1069.7951 & 1226.8210 \\
& 19 & 27.2329 & 111.0570 & 288.0516 & 595.2577 & 1069.8685 & 1555.9804 \\
& 21 & 27.2329 & 111.0570 & 288.0516 & 595.2577 & 1069.8688 & 1749.0957 \\
& Analytical* & 27.2329 & 111.0570 & 288.0516 & 595.2577 & 1069.8688 & 1749.0974 \\
\hline
\end{tabular}

* See Appendix.

beam is presented for $g / L=0.05,0.1$ and 0.2 . The buckling load converge to exact values with 8 nodes or grid points for all the three $g / L$ values. In Table 14, buckling load obtained for clamped, cantilever and propped cantilever prismatic beams are compared with analytical results given in Papargyri et al. [37] and those computed in the Appendix. The buckling load obtained using the quadrature element converges to the exact values with 11 nodes or grid points for all the boundary conditions and $g / L$ values considered.

Table 15, shows the convergence behaviour of buckling load for a simply supported beam obtained using the proposed element for sequence of $g / L$ values. Good agreement is seen for all the $g / L$ values, the buckling loads converge to analytical values using $\mathrm{N}=9$ nodes.

A simply supported tapered beam with linearly varying moment of inertia $I(x)=I_{0}\left(1-\alpha_{L}^{\chi}\right)\left(1-\gamma_{L}^{\chi}\right)^{3}$ with $0 \leq \alpha \leq 1$ and $0 \leq \gamma \leq 1$ as shown in Fig. 4, is examined for buckling analysis. Here, $\alpha$ and $\gamma$ are the degree of tapering of the width and thickness, respectively. In this study it is assumed that $\alpha=0.6$ and $\gamma=0$. In Table 16 , buckling load obtained for $g / L=$ $0.00001,0.01,0.05$ and 0.1 using the single quadrature element are shown. As the buckling load for tapered gradient beam are not available in the literature, only the classical solution [45] is compared with quadrature result obtained for $g / L=$ 0.00001. It can be seen from the tabulated results, convergence is attained with 11 nodes for all the $g / L$ values assumed. 
Table 11

Comparison of first six elastic frequencies for a free-free gradient beam.

\begin{tabular}{|c|c|c|c|c|c|c|c|}
\hline$g / L$ & $N$ & $\bar{\omega}_{1}$ & $\bar{\omega}_{2}$ & $\bar{\omega}_{3}$ & $\bar{\omega}_{4}$ & $\bar{\omega}_{5}$ & $\bar{\omega}_{6}$ \\
\hline \multirow{10}{*}{0.1} & 5 & 23.3599 & 83.6282 & 121.9217 & - & - & - \\
\hline & 7 & 23.4194 & 71.9429 & 137.0053 & 426.9289 & 452.8538 & - \\
\hline & 9 & 23.4225 & 71.7429 & 158.1114 & 311.1334 & 320.1500 & 1472.1415 \\
\hline & 11 & 23.4225 & 71.7414 & 159.5799 & 302.3470 & 421.8919 & 700.6170 \\
\hline & 13 & 23.4225 & 71.7414 & 159.5907 & 302.0790 & 510.3475 & 690.0153 \\
\hline & 15 & 23.4225 & 71.7414 & 159.5908 & 302.0760 & 516.5384 & 822.1364 \\
\hline & 17 & 23.4225 & 71.7414 & 159.5908 & 302.0760 & 516.6440 & 821.9297 \\
\hline & 19 & 23.4225 & 71.7414 & 159.5908 & 302.0759 & 516.6450 & 821.9264 \\
\hline & 21 & 23.4225 & 71.7414 & 159.5908 & 302.0759 & 516.6450 & 821.9264 \\
\hline & Analytical* & 23.4225 & 71.7414 & 159.5908 & 302.0759 & 516.6450 & 821.9264 \\
\hline \multirow{10}{*}{0.2} & 5 & 24.1794 & 98.2977 & 122.5347 & - & - & - \\
\hline & 7 & 24.8274 & 87.7257 & 147.4814 & 520.3440 & 664.4032 & - \\
\hline & 9 & 24.8322 & 87.5490 & 210.1190 & 335.6703 & 473.5781 & 2337.0770 \\
\hline & 11 & 24.8322 & 87.5481 & 221.0217 & 459.8365 & 463.2166 & 1046.2430 \\
\hline & 13 & 24.8322 & 87.5481 & 221.0947 & 462.9591 & 672.448 & 878.9483 \\
\hline & 15 & 24.8322 & 87.5481 & 221.0953 & 462.9567 & 849.7507 & 937.7545 \\
\hline & 17 & 24.8322 & 87.5481 & 221.0953 & 462.9567 & 851.7404 & 1226.7377 \\
\hline & 19 & 24.8322 & 87.5481 & 221.0953 & 459.9567 & 851.7473 & 1425.5299 \\
\hline & 21 & 24.8322 & 87.5481 & 221.0953 & 459.9567 & 851.7473 & 1425.5298 \\
\hline & Analytical* & 24.8322 & 87.5481 & 221.0953 & 459.9567 & 851.7473 & 1425.5278 \\
\hline
\end{tabular}

* See Appendix.

Table 12

Comparison of first two frequencies for a simply supported gradient beam for a sequence of $g / L$ values.

\begin{tabular}{|c|c|c|c|c|c|c|c|c|}
\hline \multirow[t]{2}{*}{$g / L$} & \multicolumn{4}{|l|}{$\omega_{1}$} & \multicolumn{4}{|l|}{$\omega_{2}$} \\
\hline & 7 & 9 & 11 & Analytical* & 7 & 9 & 11 & Analytical* \\
\hline 0.01 & 9.8662 & 9.8745 & 9.8745 & 9.8745 & 38.9263 & 39.5551 & 39.5562 & 39.5562 \\
\hline 0.03 & 9.9123 & 9.9133 & 9.9133 & 9.9133 & 40.1252 & 40.1735 & 40.1736 & 40.1736 \\
\hline 0.05 & 9.9902 & 9.9906 & 9.9906 & 9.9906 & 41.3777 & 41.3808 & 41.3808 & 41.3808 \\
\hline 0.07 & 10.1052 & 10.1054 & 10.1054 & 10.1054 & 43.1391 & 43.1282 & 43.1281 & 43.1281 \\
\hline 0.09 & 10.2564 & 10.2565 & 10.2565 & 10.2565 & 45.3717 & 45.3534 & 45.3534 & 45.3534 \\
\hline 0.10 & 10.3450 & 10.3452 & 10.3452 & 10.3452 & 46.6455 & 46.6244 & 46.6244 & 46.6244 \\
\hline 0.11 & 10.4422 & 10.4423 & 10.4423 & 10.4423 & 48.0136 & 47.9901 & 47.9900 & 47.9900 \\
\hline 0.13 & 10.6610 & 10.6610 & 10.6610 & 10.6610 & 51.0024 & 50.9744 & 50.9743 & 50.9743 \\
\hline 0.15 & 10.9105 & 10.9105 & 10.9105 & 10.9105 & 54.2811 & 54.2490 & 54.2489 & 54.2489 \\
\hline 0.17 & 11.1889 & 11.1889 & 11.1890 & 11.1890 & 57.8006 & 57.7645 & 57.7644 & 57.7644 \\
\hline 0.19 & 11.4941 & 11.4941 & 11.4941 & 11.4941 & 61.5198 & 61.4797 & 61.4796 & 61.4796 \\
\hline 0.20 & 11.6561 & 11.6561 & 11.6561 & 11.6561 & 63.4433 & 63.4012 & 63.4011 & 63.4011 \\
\hline
\end{tabular}

* See Appendix.

Table 13

Comparison of normalized buckling load for a simply supported gradient beam.

\begin{tabular}{|c|c|c|c|}
\hline \multirow[t]{2}{*}{$N$} & \multicolumn{3}{|l|}{$g / L$} \\
\hline & 0.05 & 0.1 & 0.2 \\
\hline 5 & 13.6469 & 15.1639 & 19.6774 \\
\hline 6 & 10.1480 & 10.8823 & 13.8162 \\
\hline 7 & 10.1598 & 10.8946 & 13.8319 \\
\hline 8 & 10.1132 & 10.8437 & 13.7660 \\
\hline 9 & 10.1132 & 10.8437 & 13.7660 \\
\hline 10 & 10.1131 & 10.8436 & 13.7660 \\
\hline 11 & 10.1131 & 10.8436 & 13.7660 \\
\hline Analytical [37] & 10.1131 & 10.8436 & 13.7660 \\
\hline FE-Exact[41] & 10.1131 & 10.8436 & 13.7660 \\
\hline
\end{tabular}


Table 14

Comparison of normalized buckling load for a clamped, cantilever and propped-cantilever gradient beams.

\begin{tabular}{|c|c|c|c|c|c|c|}
\hline \multirow[t]{2}{*}{$N$} & \multicolumn{2}{|l|}{ Clamped } & \multicolumn{2}{|l|}{ Cantilever } & \multicolumn{2}{|c|}{ Propped cantilever } \\
\hline & 0.1 & 0.2 & 0.1 & 0.2 & 0.1 & 0.2 \\
\hline 5 & 151.9976 & 236.0564 & 3.0986 & 4.0248 & 47.7472 & 84.1677 \\
\hline 7 & 80.4753 & 199.6620 & 3.0996 & 3.9701 & 30.1430 & 54.1145 \\
\hline 9 & 83.2963 & 197.9525 & 3.1012 & 3.9703 & 29.8796 & 53.3654 \\
\hline 11 & 83.2909 & 197.9570 & 3.1013 & 3.9703 & 29.8777 & 53.3594 \\
\hline 13 & 83.2917 & 197.9570 & 3.1013 & 3.9703 & 29.8777 & 53.3594 \\
\hline 15 & 83.2917 & 197.9570 & 3.1013 & 3.9703 & 29.8777 & 53.3594 \\
\hline 17 & 83.2917 & 197.9570 & 3.1013 & 3.9703 & 29.8777 & 53.3594 \\
\hline 19 & 83.2917 & 197.9570 & 3.1013 & 3.9703 & 29.8777 & 53.3594 \\
\hline Analytical* & 83.2917 & 197.9570 & $3.1013[40]$ & $3.9703[40]$ & 29.8777 & 53.3594 \\
\hline
\end{tabular}

* See Appendix.

\section{Table 15}

Comparison of normalized buckling load for a simply supported beam for a sequence of $g / L$ values.

\begin{tabular}{llllll}
\hline$g / L$ & $N$ & & & \\
\cline { 2 - 6 } & 5 & 7 & 9 & 11 & Analytical $^{*}$ \\
\hline 0.01 & 6.7933 & 9.9205 & 9.8745 & 9.8793 & 9.8793 \\
0.03 & 12.5301 & 10.0027 & 9.9573 & 9.9573 & 9.9573 \\
0.05 & 13.6469 & 10.1598 & 10.1132 & 10.1131 & 10.1131 \\
0.07 & 14.2664 & 10.3950 & 10.3470 & 10.3469 & 10.3469 \\
0.09 & 14.8507 & 10.7085 & 10.6587 & 10.6586 & 10.6586 \\
0.10 & 15.1639 & 10.8946 & 10.8437 & 10.8437 & 10.8437 \\
0.11 & 15.4980 & 11.1003 & 11.0483 & 11.0482 & 11.0482 \\
0.13 & 16.2382 & 11.5703 & 11.5159 & 11.5158 & 11.5158 \\
0.15 & 17.0829 & 12.1186 & 12.0614 & 12.0613 & 12.0613 \\
0.17 & 18.0369 & 12.7452 & 12.6848 & 12.6847 & 12.6847 \\
0.19 & 19.1025 & 13.4501 & 13.3861 & 13.3861 & 13.3861 \\
0.20 & 19.6774 & 13.8319 & 13.7660 & 13.7660 & 13.7660 \\
\hline
\end{tabular}

* See Appendix.

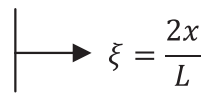

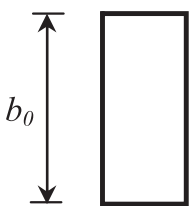

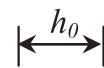

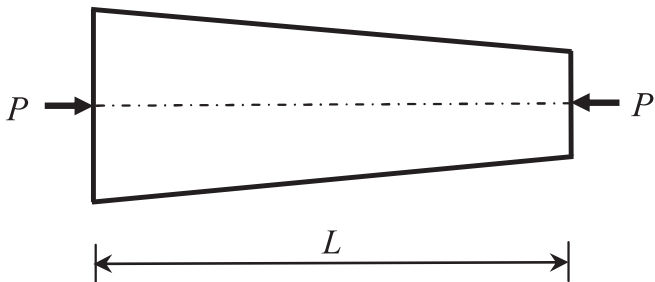

Fig. 4. A linearly varying simply supported gradient beam.

Table 16

Comparison of normalized buckling load for a tapered simply supported gradient beam (Tapering angles: $\alpha=0.6, \gamma=0$ ).

\begin{tabular}{|c|c|c|c|c|}
\hline \multirow[t]{2}{*}{$N$} & \multicolumn{4}{|l|}{$g / L$} \\
\hline & 0.00001 & 0.01 & 0.05 & 0.1 \\
\hline 7 & 6.6656 & 6.6696 & 6.8345 & 7.3427 \\
\hline 9 & 6.6792 & 6.6695 & 6.8491 & 7.3552 \\
\hline 11 & 6.6787 & 6.6856 & 6.8489 & 7.3551 \\
\hline 13 & 6.6787 & 6.6856 & 6.8489 & 7.3551 \\
\hline 15 & 6.6787 & 6.6856 & 6.8489 & 7.3551 \\
\hline 17 & 6.6787 & 6.6856 & 6.8489 & 7.3551 \\
\hline 19 & 6.6787 & 6.6856 & 6.8489 & 7.3551 \\
\hline 21 & 6.6787 & 6.6856 & 6.8489 & 7.3551 \\
\hline Ref. [45] $(g / L=0)$ & 6.675 & - & - & - \\
\hline
\end{tabular}

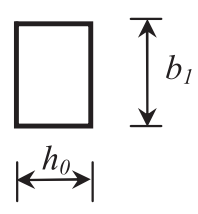


Hence, from the above observations it can be concluded that the proposed element can be efficiently applied for buckling analysis of gradient elastic prismatic and non-prismatic beams for different boundary conditions and $g / L$ values.

\section{Conclusion}

A novel weak form quadrature element was proposed for non-classical strain gradient Euler-Bernoulli beam theory. The proposed element has displacement as the only degree of freedom in the element domain and displacement, slope and curvature at the boundaries. All the classical and non-classical boundary conditions associated with the gradient beam theory were represented accurately and with ease. The capability of the element was demonstrated through numerical examples on bending, free vibration and stability analysis of prismatic and non-prismatic gradient beams. Based on the findings it was concluded that, the quadrature element with reasonable number of nodes can produce accurate solutions for static, free vibration and buckling analysis of gradient elastic beams for various boundary conditions and length scale parameters.

\section{Acknowledgement}

The authors would like to thank Professor Xinwei Wang, Nanjing University of Aeronautics and Astronautics, People's Republic of China, for the useful technical discussions.

\section{Appendix A. Analytical solutions for static analysis of gradient elastic Euler-Bernoulli beam:}

To obtain the static deflections of the gradient elastic Euler-Bernoulli beam which is governed by Eq. (8), we assume a solution of the form

$$
w(x)=\tilde{c}_{1} x^{3}+\tilde{c}_{2} x^{2}+\tilde{c}_{3} x+\tilde{c}_{4}+\tilde{c}_{5} g^{4} \sinh (x / g)+\tilde{c}_{6} g^{4} \cosh (x / g)-(q / 24 E I) x^{4}
$$

The constants $\tilde{c}_{1}-\tilde{c}_{6}$ are determined with the aid of boundary conditions listed in Eqs. (9) and (10). After applying the boundary conditions the system of equations are expressed as:

$$
[\tilde{K}]\{\delta\}=\{f\}
$$

where $\tilde{K}$ is the coefficient matrix, $f$ is the vector corresponding to the load and $\{\delta\}=\left\{\tilde{c}_{1}, \tilde{c}_{2}, \tilde{c}_{3}, \tilde{c}_{4}, \tilde{c}_{5}, \tilde{c}_{6}\right\}$ is the unknown constant vector to be determined. Once the unknown constants are determined then the displacement solution is obtained from Eq. (A.1). The slope and curvature at any point along the length of the beam can be obtained by performing the first and second derivatives of the deflection. The shear force, bending moment and higher moment are obtained by substituting Eq. (A.1) in Eqs. (9) and (10). The following are the expressions for support reactions:

$$
\begin{aligned}
& \text { Shearforce }: V=6 E I \tilde{c}_{1}-\frac{q x}{E I} \\
& \text { Bendingmoment }: M=2 E I\left(\tilde{c}_{2}+3 \tilde{c}_{1} x\right)+\frac{q}{E I}\left[g^{2}-\frac{x^{2}}{2}\right] \\
& \text { Highermoment }: \bar{M}=6 E \operatorname{Ig}^{2} \tilde{c}_{1}+E \operatorname{Ig}^{3} \cosh (x / g) \tilde{c}_{5}+\operatorname{EIg}^{3} \sinh (x / g) \tilde{c}_{6}-q g^{2} x
\end{aligned}
$$

The following are the list of simultaneous equations to determine the unknown constants for different boundary conditions:

(a) Simply supported beam :

$$
[\tilde{K}]=\left[\begin{array}{cccccc}
0 & 0 & 0 & 1 & 0 & g^{4} \\
0 & 2 & 0 & 0 & 0 & 0 \\
L^{3} & L^{2} & L & 1 & g^{4} \sinh (L / g) & g^{4} \cosh (L / g) \\
6 L & 2 & 0 & 0 & 0 & 0 \\
0 & 2 & 0 & 0 & 0 & g^{2} \\
6 L & 2 & 0 & 0 & g^{2} \sinh (L / g) & g^{2} \cosh (L / g)
\end{array}\right],\{f\}=\left\{\begin{array}{c}
0 \\
g^{2} q / E I \\
-q L^{4} / 24 E I \\
g^{2} q / E I-q L^{2} / 2 E I \\
0 \\
-q L^{2} / 2 E I
\end{array}\right\}
$$

(b) Pinned-guided beam :

$$
[\tilde{K}]=\left[\begin{array}{cccccc}
0 & 0 & 0 & 1 & 0 & g^{4} \\
0 & 2 & 0 & 0 & 0 & 0 \\
0 & 2 & 0 & 0 & 0 & g^{2} \\
6 & 0 & 0 & 0 & 0 & 0 \\
3 L^{2} & 2 L & 1 & 0 & g^{3} \cosh (L / g) & g^{3} \sinh (L / g) \\
6 & 0 & 0 & 0 & g \cosh (L / g) & g \sinh (L / g)
\end{array}\right],\{f\}=\left\{\begin{array}{c}
0 \\
g^{2} q / E I \\
0 \\
-q L / E I \\
-q L^{3} / 6 E I \\
-q L / E I
\end{array}\right\}
$$


Appendix B. Analytical solutions for free vibration analysis of gradient elastic Euler-Bernoulli beam:

To obtain the natural frequencies of the gradient elastic Euler-Bernoulli beam which is governed by Eq. (8), we assume a solution of the form

$$
w(x, t)=\bar{w}(x) e^{i \omega t}
$$

substituting the above solution in the governing equation Eq. (8), we get

$$
\bar{w}^{i v}-g^{2} \bar{w}^{v i}-\frac{\omega^{2}}{\beta^{2}} \bar{w}=0
$$

here, $\beta^{2}=E I / m$, and the above equation has the solution of type

$$
\bar{w}(x)=\sum_{j=1}^{6} \bar{c}_{i} e^{k_{i} x}
$$

where $\bar{c}_{i}$ are the constants of integration which are determined through boundary conditions and the $k_{i}$ are the roots of the characteristic equation

$$
k^{i v}-g^{2} k^{v i}-\frac{\omega^{2}}{\beta^{2}}=0
$$

after applying the boundary conditions listed in Section 2 we get

$$
[F(\omega)]\{\bar{c}\}=\{0\}
$$

for non-trivial solution, following condition should be satisfied

$$
\operatorname{det}[F(\omega)]=0
$$

The above frequency equation renders all the natural frequencies for a gradient elastic Euler-Bernoulli beam. The following are the frequency equations for different boundary conditions.

(a) Cantilever beam :

$$
[F(\omega)]=\left[\begin{array}{cccccc}
1 & 1 & 1 & 1 & 1 & 1 \\
k_{1} & k_{2} & k_{3} & k_{4} & k_{5} & k_{6} \\
k_{1}{ }^{2} & k_{2}{ }^{2} & k_{3}{ }^{2} & k_{4}{ }^{2} & k_{5}{ }^{2} & k_{6}{ }^{2} \\
t_{1} & t_{2} & t_{3} & t_{4} & t_{5} & t_{6} \\
p_{1} & p_{2} & p_{3} & p_{4} & p_{5} & p_{6} \\
k_{1}^{3} e^{\left(k_{1} L\right)} & k_{2}^{3} e^{\left(k_{2} L\right)} & k_{3}^{3} e^{\left(k_{3} L\right)} & k_{4}^{3} e^{\left(k_{4} L\right)} & k_{5}^{3} e^{\left(k_{5} L\right)} & k_{6}^{3} e^{\left(k_{6} L\right)}
\end{array}\right]
$$

(b) Propped cantilever beam :

$$
[F(\omega)]=\left[\begin{array}{cccccc}
1 & 1 & 1 & 1 & 1 & 1 \\
k_{1} & k_{2} & k_{3} & k_{4} & k_{5} & k_{6} \\
k_{1}{ }^{2} & k_{2}{ }^{2} & k_{3}{ }^{2} & k_{4}{ }^{2} & k_{5}{ }^{2} & k_{6}{ }^{2} \\
e^{\left(k_{1} L\right)} & e^{\left(k_{2} L\right)} & e^{\left(k_{3} L\right)} & e^{\left(k_{4} L\right)} & e^{\left(k_{5} L\right)} & e^{\left(k_{6} L\right)} \\
k_{1}^{2} e^{\left(k_{1} L\right)} & k_{2}^{2} e^{\left(k_{2} L\right)} & k_{3}^{2} e^{\left(k_{3} L\right)} & k_{4}^{2} e^{\left(k_{4} L\right)} & k_{5}^{2} e^{\left(k_{5} L\right)} & k_{6}^{2} e^{\left(k_{6} L\right)} \\
p_{1} & p_{2} & p_{3} & p_{4} & p_{5} & p_{6}
\end{array}\right]
$$

(d) Free-free beam :

$$
[F(\omega)]=\left[\begin{array}{cccccc}
q_{1} & q_{2} & q_{3} & q_{4} & q_{5} & q_{6} \\
r_{1} & r_{2} & r_{3} & r_{4} & r_{5} & r_{6} \\
k_{1}^{3} & k_{2}^{3} & k_{3}^{3} & k_{4}^{3} & k_{5}^{3} & k_{6}^{3} \\
t_{1} & t_{2} & t_{3} & t_{4} & t_{5} & t_{6} \\
p_{1} & p_{2} & p_{3} & p_{4} & p_{5} & p_{6} \\
k_{1}^{3} e^{\left(k_{1} L\right)} & k_{2}^{3} e^{\left(k_{2} L\right)} & k_{3}^{3} e^{\left(k_{3} L\right)} & k_{4}^{3} e^{\left(k_{4} L\right)} & k_{5}^{3} e^{\left(k_{5} L\right)} & k_{6}^{3} e^{\left(k_{6} L\right)}
\end{array}\right]
$$

Where

$$
\begin{array}{lll}
t_{1}=\left(k_{1}^{3}-g^{2} k_{1}{ }^{5}\right) e^{\left(k_{1} L\right)}, & t_{2}=\left(k_{2}^{3}-g^{2} k_{2}{ }^{5}\right) e^{\left(k_{2} L\right)}, & t_{3}=\left(k_{3}^{3}-g^{2} k_{3}{ }^{5}\right) e^{\left(k_{3} L\right)} \\
t_{4}=\left(k_{4}^{3}-g^{2} k_{4}{ }^{5}\right) e^{\left(k_{4} L\right)}, & t_{5}=\left(k_{5}^{3}-g^{2} k_{5}^{5}\right) e^{\left(k_{5} L\right)}, & t_{6}=\left(k_{6}^{3}-g^{2} k_{6}{ }^{5}\right) e^{\left(k_{6} L\right)} \\
p_{1}=\left(k_{1}^{2}-g^{2} k_{1}{ }^{4}\right) e^{\left(k_{1} L\right)}, & p_{2}=\left(k_{2}^{2}-g^{2} k_{2}{ }^{4}\right) e^{\left(k_{2} L\right)}, & p_{3}=\left(k_{3}^{2}-g^{2} k_{3}{ }^{4}\right) e^{\left(k_{3} L\right)} \\
p_{4}=\left(k_{4}^{2}-g^{2} k_{4}{ }^{4}\right) e^{\left(k_{4} L\right)}, & p_{5}=\left(k_{5}^{2}-g^{2} k_{5}{ }^{4}\right) e^{\left(k_{5} L\right)}, & p_{6}=\left(k_{6}^{2}-g^{2} k_{6}{ }^{4}\right) e^{\left(k_{6} L\right)}
\end{array}
$$




$$
\begin{array}{lll}
q_{1}=\left(k_{1}^{3}-g^{2} k_{1}{ }^{5}\right), & q_{2}=\left(k_{2}^{3}-g^{2} k_{2}{ }^{5}\right), & q_{3}=\left(k_{3}^{3}-g^{2} k_{3}{ }^{5}\right) \\
q_{4}=\left(k_{4}^{3}-g^{2} k_{4}{ }^{5}\right), & q_{5}=\left(k_{5}^{3}-g^{2} k_{5}{ }^{5}\right), & q_{6}=\left(k_{6}^{3}-g^{2} k_{6}{ }^{5}\right) \\
r_{1}=\left(k_{1}^{2}-g^{2} k_{1}{ }^{4}\right), & r_{2}=\left(k_{2}^{2}-g^{2} k_{2}{ }^{4}\right), & r_{3}=\left(k_{3}^{2}-g^{2} k_{3}{ }^{4}\right) \\
r_{4}=\left(k_{4}^{2}-g^{2} k_{4}{ }^{4}\right), & r_{5}=\left(k_{5}^{2}-g^{2} k_{5}{ }^{4}\right), & r_{6}=\left(k_{6}^{2}-g^{2} k_{6}{ }^{4}\right)
\end{array}
$$

\section{Appendix C. Analytical solutions for buckling analysis of gradient elastic Euler-Bernoulli beam:}

To obtain the buckling load of the gradient elastic Euler-Bernoulli beam which is governed by Eq. (8), we assume a solution of the form

$$
w(x)=d_{1}+d_{2} x+d_{3} \sin \left(\alpha_{1} x\right)+d_{4} \cos \left(\alpha_{1} x\right)+d_{5} \sinh \left(\alpha_{2} x\right)+d_{6} \cosh \left(\alpha_{2} x\right)
$$

where $d_{i}$ are the constants of integration which are determined through boundary conditions and the $\alpha_{1}$ and $\alpha_{2}$ are the roots of the characteristic equation given by

$$
\alpha_{1}=\left(\frac{-1+\sqrt{1+4 g^{2} P / E I}}{2 g^{2}}\right)^{\frac{1}{2}}, \quad \alpha_{2}=\left(\frac{1+\sqrt{1+4 g^{2} P / E I}}{2 g^{2}}\right)^{\frac{1}{2}}
$$

After applying the boundary conditions listed in Section 2 we get

$$
[\bar{G}(P)]\{d\}=\{0\}
$$

For non-trivial solution, following condition should be satisfied

$$
\operatorname{det}[\bar{G}(P)]=0
$$

The above Eigenvalue problem renders the buckling load for a gradient elastic Euler-Bernoulli beam. The following are the system equations for different boundary conditions.

(a) clamped beam :

$$
[\bar{G}(P)]=\left[\begin{array}{cccccc}
1 & 0 & 0 & 1 & 0 & 1 \\
0 & 1 & \alpha_{1} & 0 & \alpha_{2} & 0 \\
0 & 0 & 0 & -\alpha_{1}^{2} & 0 & -\alpha_{2}^{2} \\
1 & L & \sin \left(\alpha_{1} L\right) & \cos \left(\alpha_{1} L\right) & \sinh \left(\alpha_{2} L\right) & \cosh \left(\alpha_{2} L\right) \\
0 & 1 & \alpha_{1} \cos \left(\alpha_{1} L\right) & -\alpha_{1} \sin \left(\alpha_{1} L\right) & \alpha_{2} \cosh \left(\alpha_{2} L\right) & -\alpha_{2} \sinh \left(\alpha_{2} L\right) \\
0 & 0 & -\alpha_{1}^{2} \sin \left(\alpha_{1} L\right) & -\alpha_{1}^{2} \cos \left(\alpha_{1} L\right) & \alpha_{2}^{2} \sinh \left(\alpha_{2} L\right) & \alpha_{2}^{2} \cosh \left(\alpha_{2} L\right)
\end{array}\right]
$$

(b) Propped cantilever beam :

$$
[\bar{G}(P)]=\left[\begin{array}{cccccc}
1 & 0 & 0 & 1 & 0 & 1 \\
0 & 1 & \alpha_{1} & 0 & \alpha_{2} & 0 \\
0 & 0 & 0 & -\alpha_{1}^{2} & 0 & -\alpha_{2}^{2} \\
1 & L & \sin \left(\alpha_{1} L\right) & \cos \left(\alpha_{1} L\right) & \sinh \left(\alpha_{2} L\right) & \cosh \left(\alpha_{2} L\right) \\
0 & 1 & \alpha_{1} \cos \left(\alpha_{1} L\right) & -\alpha_{1} \sin \left(\alpha_{1} L\right) & \alpha_{2} \cosh \left(\alpha_{2} L\right) & -\alpha_{2} \sinh \left(\alpha_{2} L\right) \\
0 & 0 & -\alpha_{1}^{2} \sin \left(\alpha_{1} L\right) & -\alpha_{1}^{2} \cos \left(\alpha_{1} L\right) & \alpha_{2}^{2} \sinh \left(\alpha_{2} L\right) & \alpha_{2}^{2} \cosh \left(\alpha_{2} L\right)
\end{array}\right]
$$

\section{References}

[1] O.C. Zienkiewicz, R.L. Taylor, The Finite Element Method, Vol.1. Basic Formulations and Linear Problems, McGraw-Hill, London, 1989 , p. p.648.

[2] O.C. Zienkiewicz, R.L. Taylor, The Finite Element Method, Vol.2. Solid and Fluid Mechanics: Dynamics and Non-linearity, McGraw-Hill, London, 1991, p. p.807.

[3] K.J. Bathe, Finite Element Procedures in Engineering Analysis, Prentice-Hall, 1982.

[4] G.D. Smith, Numerical Solution of Partial Differential Equations: Finite Difference Methods, Clarendon press, Oxford, 1985.

[5] J.C. Strikwerda, Finite Difference Schemes and Partial Differential Equations, Chapman \& Hall, New York, 1990.

[6] X. Wang, Differential Quadrature and Differential Quadrature Based Element Methods Theory and Applications, Elsevier, USA, 2015.

[7] R.E. Bellman, J. Casti, Differential quadrature and long-term integration, J. Math. Anal. Appl. 34 (1971) 235-238.

[8] G. Karami, P. Malekzadeh, A new differential quadrature methodology for beam analysis and the associated differential quadrature element method, Comput. Methods Appl. Mech. Eng. 191 (2002) 3509-3526.

[9] C.W. Bert, M. Malik, Differential quadrature method in computational mechanics: a review, ASME Appl. Mech. Rev. 49 (1) (1996) 1-28.

[10] C.W. Bert, M. Malik, The differential quadrature method for irregular domains and application to plate vibration, Int. J. Mech. Sci. 199638 (1996) 589-606.

[11] C. Shu, Differential Quadrature and Its Application in Engineering, Springer-Verlag, London, 2000.

[12] H. Du, M.K. Lim, N.R. Lin, Application of generalized differential quadrature method to structural problems, Int. J. Num. Methods Eng. 37 (1994) 1881-1896.

[13] O. Civalek, Application of differential quadrature and harmonic differential quadrature for buckling analysis of thin isotropic plates and elastic columns, Eng. Struct. 26 (2) (2004) 171-186.

[14] X. Wang, H.Z. Gu, Static analysis of frame structures by the differential quadrature element method, Int. J. Numer. Methods Eng. 40 (1997) 759-772. 
[15] Y. Wang, X. Wang, Y. Zhou, Static and free vibration analyses of rectangular plates by the new version of differential quadrature element method, Int. J. Numer. Method Eng. 59 (2004) 1207-1226.

[16] Y. Wang, Y.B. Zhao, G.W. Wei, A note on the numerical solution of high-order differential equations, J. Comput. Appl. Math. 159 (2003) 387-398.

[17] T.Y. Wu, G.R. Liu, Application of the generalized differential quadrature rule to sixth-order differential equations, Commun. Numer. Methods Eng. 16 (2000) 777-784.

[18] G.R. Liu, T.Y. Wu, Differential quadrature solutions of eighth-order boundary-value differential equations, J. Comput. Appl. Math. 145 (2002) 223-235.

[19] X. Wang, Z. Yuan, C. Jin, Weak form quadrature element method and its applications in science and engineering: a state-of-the-art review, Appl. Mech. Rev. 69 (3) (2017) 030801.

[20] H. Zhong, T. Yu, Flexural vibration analysis of an eccentric annular Mindlin plate, Arch. Appl. Mech. 77 (4) (2007) $185-195$.

[21] H. Zhong, T. Yu, A weak form quadrature element method for plane elasticity problems, Appl. Math. Model. 33 (2009) $3801-3814$.

[22] H. Zhong, M. Gao, Quadrature element analysis of planar frameworks, Arch. Appl. Mech. 80 (12) (2010) $1391-1405$.

[23] H. Zhong, Y. Wang, Weak form quadrature element analysis of Bickford beams, Eur. J. Mech./Solids 29 (5) (2010) 851-858.

[24] H. Zhong, R. Zhang, H. Yu, Buckling analysis of planar frameworks using the quadrature element method, 11, Int. J. Struct. Stab. Dyn. 11 (2) (2011).

[25] H.Z. Zhong, Z.G. Yue, Analysis of thin plates by the weak form quadrature element method, Sci. China Phys. Mech. 55 (5) (2012) 861-871.

[26] C. Jin, X. Wang, L. Ge, Novel weak form quadrature element method with expanded Chebyshev nodes, Appl. Math. Lett. 34 (2014) 51-59.

[27] X. Wang, Z. Yuan, Techniques for vibration analysis of hybrid beam and ring structures with variable thickness, Comput. Struct. 206 (2018) $109-121$.

[28] M. Liao, F. Chen, Z. Chen, Y.B. Yang, A weak-form quadrature element formulation for 3d beam elements used in nonlinear and postbuckling analysis of space frames, Eng. Struct. 145 (2017) 34-43.

[29] M. Ishaquddin, S. Gopalakrishnan, Novel weak form quadrature elements for non-classical higher order beam and plate theories, http://arxiv.org/abs/ 1802.05541.

[30] R. Mindlin, N. Eshel, On first strain-gradient theories in linear elasticity, Int. J. Solids Struct. 4 (1968) 109-124.

[31] N.A. Fleck, J.W. Hutchinson, A phenomenological theory for strain gradient effects in plasticity, J. Mech. Phys. Solids 41 (12) (1993) $1825-1857$.

[32] W.T. Koiter, Couple-stresses in the theory of elasticity, I \& II, Proc. K. Ned. Akad. Wet. (B) 67 (1964) 17-44.

[33] H. Askes, E.C. Aifantis, Gradient elasticity in statics and dynamics: An overview of formulations, length scale identification procedures, finite element implementations and new results, Int. J. Solids Struct. 48 (2011) 1962-1990.

[34] R.D. Mindlin, Micro-structure in linear elasticity, Arch. Rat. Mech. Anal. 16 (1964) 52-78.

[35] E.C. Aifantis, Update on a class of gradient theories, Mech. Mater. 35 (2003) 259-280.

[36] B.S. Altan, E.C. Aifantis, On some aspects in the special theory of gradient elasticity, J. Mech. Behav. Mater. 8 (3) (1997) $231-282$.

[37] S. Papargyri-Beskou, K.G. Tsepoura, D. Polyzos, D.E. Beskos, Bending and stability analysis of gradient elastic beams, Int. J. Solids Struct. 40 (2003) $385-400$.

[38] S. Papargyri-Beskou, D. Polyzos, D.E. Beskos, Dynamic analysis of gradient elastic flexural beams, Struct. Eng. Mech. 15 (6) (2003) $705-716$.

[39] A.K. Lazopoulos, Dynamic response of thin strain gradient elastic beams, Int. J. Mech. Sci. 58 (2012) 27-33.

[40] K.A. Lazopoulos, A.K. Lazopoulos, Bending and buckling of thin strain gradient elastic beams, Eur. J. Mech. A/Solids 29 (2010) 837-843.

[41] I.P. Pegios, S. Papargyri-Beskou, D.E. Beskos, Finite element static and stability analysis of gradient elastic beam structures, Acta Mech 226 (2015) $745-768$.

[42] I. Vardoulakis, J. Sulem, Bifurcation Analysis in Geomechanics, Blackie/Chapman and Hall, London, 1995.

[43] J.N. Reddy, Energy Principles and Variational Methods in Applied Mechanics, second edition, John Wiley, NY, 2002.

[44] M. Kitahara, Boundary Integral Equation Methods in Eigenvalue Problems of Elastodynamics and Thin Plates, Elsevier, Amsterdam, 1985.

[45] A. Nikolic, S. Salinic, Buckling analysis of non-prismatic columns: a rigid multibody approach, Eng. Struct. 143 (2017) $511-521$. 